\title{
Vibration damping of the anti-vibration platform intended for use in combination with audio/music devices
}

\author{
Lukasz Breńkacz ${ }^{1}$, Pawel Bagiński², Jarosław K. Korbicz ${ }^{3}$ \\ ${ }^{1,2}$ Institute of Fluid Flow Machinery, Polish Academy of Sciences, Gdansk, Poland \\ ${ }^{3}$ STACORE, Kartuska 4, 80-297 Miszewo, Gdansk, Poland \\ ${ }^{1}$ Corresponding author \\ E-mail: ${ }^{1}$ lbrenkacz@imp.gda.pl, ${ }^{2}$ pbaginski@imp.gda.pl, ${ }^{3}$ info@stacore.pl \\ Received 5 June 2019; received in revised form 15 October 2019; accepted 27 October 2019 \\ DOI https://doi.org/10.21595/jve.2019.20846
}

Check for updates

Copyright $(2020$ Eukasz Breńkacz, et al. This is an open access article distributed under the Creative Commons Attribution License, which permits unrestricted use, distribution, and reproduction in any medium, provided the original work is properly cited.

\begin{abstract}
The article presents research on the damping properties of an anti-vibration platform (designed and manufactured by Stacore), which is intended for use in combination with high class audio devices. The platform comprises two parts that are capable of passive vibration damping. The design of this platform is unique and has been developed by applying several technical solutions in a combination not found on any other anti-vibration platform on the audio market. These solutions are described in the article. The first part of the platform is pneumatically operated and the second part uses ball bearings. The casing also fulfils the most important function vibration damping. It is made of amorphous slate (known for its good vibration-damping properties) and includes a metal plate covered with a special visco-elastic layer that fulfils the role of binding material. The first part of the platform realises the pneumatic damping. It comprises four elastomer pneumatic springs, each with its own air tank. The air tanks are designed to operate at a maximum pressure of 5 bar. The second vibration-damping part of the platform is located above the first part (pneumatic) and consists of ball bearings, used for the isolation of transverse vibration, being relatively difficult to deal with pneumatic springs. The upper part consists of three bearings, each of which comprises of a polished, deep hardened steel racings and a ball made of tungsten carbide. The scientific literature describes many solutions enabling vibration damping, including many anti-vibration platforms. However, the literature lacks the descriptions of analyses conducted on anti-vibration platforms for audio devices. This article is a novelty in the literature as it concerns the experimental research aimed at verifying the vibration-damping capacity of the anti-vibration platform that can be used with audio devices owned by people who appreciate high-quality music. The article describes in detail the whole measurement procedure applied to the vibration damping platform. For research purposes, the anti-vibration platform was suspended on flexible ropes. At first, an electromagnetic vibration exciter was attached to the base on which the platform rested, and then the displacements of the upper and lower part of the platform were measured using laser sensors. Based on these signals, the vibration damping capability (transmissibility) of the platform was determined in two mutually perpendicular directions. In addition to the graph that shows the vibration damping capability of the anti-vibration platform, the signals of the applied force and displacements measured during the research are also presented in this article.
\end{abstract}

Keywords: anti-vibration platform, vibration damping in audio devices, experimental measurement.

\section{Introduction}

Vibration reduction in a variety of situations improves quality of life and safety. In the literature, there are many articles on vibration damping for a wide range of applications. The literature review provides information on industrial branches in which it is necessary to dampen vibrations. Next, this article focuses on anti-vibration platforms, paying particular attention to the 
reduction of vibrations in audio systems.

In everyday life, there are many situations where vibrations are a problem. The situation described in article [1] by Paolo Clemente and Dario Rinaldis is an example. They looked into the problem of protecting buildings from traffic vibrations. This is an interesting example because such vibrations can also be transmitted to audio sets (which can be used, for example, in combination with the anti-vibration platform which is a research object in this paper). The authors of article [1] committed themselves to protect an ancient building in Rome against vibrations coming from road traffic. To do this, an anti-vibration system was placed under the pavement of a nearby road. The propagation of waves in the soil around the building with and without the presence of the anti-vibration system was studied. It turned out that the anti-vibration system was able to reduce vibrations caused by road traffic. In the summary of the article, the authors point out that contemporary streets are of fairly good quality and the amplitude of vibrations coming from road traffic is low.

The measurement and assessment of hand-transmitted vibrations are presented by Griffin in his article [2]. The author presented the measurement methods used and discussed the obtained results. According to him, it is not necessary to measure vibrations whose frequencies can reach $1,000 \mathrm{~Hz}$. It is probably enough to limit vibration measurements to a frequency range in which the upper bound is lower than $250 \mathrm{~Hz}$. The author believes that when analysing the influence of low-frequency vibrations on the human body, frequencies below $20 \mathrm{~Hz}$ should not be taken into account, especially when analysing the effect of vibrations on blood vessels. The lower limit value greatly affects the design and evaluation of anti-vibration devices or personal protective equipment, such as gloves. The author explains that the mechanism of damage is unknown, so it is not surprising that the methods of predicting the effects of vibrations are characterised by a very high degree of uncertainty. Żywica et al. [3] stated that the transmission of vibrations in complex systems could be a difficult task. There can be a lot of dependencies.

Hand-transmitted vibrations when working with certain tools are an example of vibrations affecting the human body. Article [4] describes the research carried out on a group of forestry workers exposed to saw vibrations. It was shown that the use of anti-vibration saws had a beneficial effect on the symptoms of finger blanching. Anti-vibration gloves are another example of a tool that can reduce hand-transmitted vibrations. S. Hewitt et al. presented such gloves in the paper. Anti-vibration gloves were used to block the transmission of vibration from powered hand tools to the user, and to protect users from the negative health consequences associated with vibration exposure. The authors point out that some countries have adopted standards and/or regulations to help control exposure to hand-transmitted vibration. They believe that even though the ISO 10819 standard defines a method for testing and evaluating anti-vibration gloves, a glove certified as an anti-vibration glove by one laboratory may not pass the same test in other laboratories. The authors have shown that the effectiveness of vibration damping of anti-vibration gloves depends not only on the gloves themselves but also on many other factors such as tools, materials, vibration direction and grip force. Research findings have shown that anti-vibration gloves cannot reduce the exposure to vibrations caused by low-frequency tools such as rammers or pavement tampers. In the case of the vast majority of powered hand tools or machines such as chipping hammers, rock drills, riveting hammers and grinders, anti-vibration gloves can slightly reduce the frequency-weighted vibration transmitted to the palm of the hand and reduce little of the vibrations transmitted to the fingers. If the tools mainly generate high-frequency vibrations (above $250 \mathrm{~Hz}$ ), anti-vibration gloves can significantly reduce hand-transmitted vibrations. However, such cases are very rare. Moreover, anti-vibration gloves can have adverse effects, such as increased grip strength and reduced manual dexterity. Krajnak et al. wrote an article [5] on the effects of anti-vibration gloves on vascular and sensorineural function using an animal model. They studied the effectiveness of vibration damping of the anti-vibration material (used for the manufacture of anti-vibration gloves). The material was placed between the rat's tail and the platform used to generate vibrations. The purpose of the research was to determine if anti-vibration materials reduce the effects of vibrations on physiological symptoms such as vascular and 
peripheral nerve injury. The rats were exposed to tail vibrations for a period of four hours. The animals were tested for tactile sensitivity to the pressure applied both before and after the exposure to vibrations. It was found that the rats that were exposed to vibrations were less sensitive to pressure the day after exposure than rats not exposed to vibrations, regardless of whether or not the anti-vibration materials were used. In conclusion, the authors state that anti-vibration gloves may not offer protection against the adverse health consequences of the exposure to vibrations under all conditions.

Many scientific publications are devoted to the potential risks of vibrations on human health. Il-Kyu Hwang and Kyu-Jung Kim carried out research [6] on a commercial wrist guard to study its vibration damping capacity. They analysed wrist guards with four different padding conditions, including, but not limited to, viscoelastic polymer padding and air cell padding. Wrist injuries were tested by analysing the impact force attenuation and the maximum energy absorption of the ground reaction force measured under the simulated impact of the forearm. It turned out that additional padding on the bare hand could improve the maximum energy absorption by more than $39 \%$.

Passive and active vibration damping systems are very often used to control vehicle vibrations. These systems are constantly being developed and improved, and their parameters are being adapted to new car designs. One of the articles [7] proposes sensorless vibration control for semi-active suspensions. A semi-active suspension model, including a filtered feedback scheme and actuator dynamics, was built for research purposes. Furthermore, a direct fuzzy compensation scheme with genetic optimisation was used.

Many devices must have good vibration-damping properties to allow people to use them easily. Paper [8] describes a vibration reduction algorithm applied to the system used to detect small objects at sea. Stiffness and damping coefficients are in many cases the most important parameters used to estimate the dynamic performance of systems $[9,10]$. By knowing the values of stiffness and damping parameters, these systems can be optimised to achieve desired results $[11,12]$.

\subsection{Anti-vibration platforms}

There are many anti-vibration platforms adapted to damping vibrations of various types. Nevertheless, there are no scientific articles on the vibration damping efficiency of anti-vibration platforms specifically designed for use in combination with audio/music systems. Due to the considerable similarities between the anti-vibration platform for audio systems and other types of anti-vibration platforms, this part of the article presents a brief literature review of the most common designs of anti-vibration platforms.

In article [13], Xianglong Wen et al. presented a platform designed for vibration damping, equipped with a truss-type raft frame (made of carbon fibre-reinforced plastic). A numerical model of the frame was created. In addition, the experimental research was performed, during which a motor was placed on the platform. The motor was supported by anti-vibration footers and the truss-type frame was supported by pneumatic springs. The whole system rested on a pedestal, the vibrations of which were forced by means of an electromagnetic vibration exciter. Damping of the developed construction was determined. Furthermore, a FEM model was built using the ABAQUS software to evaluate the vibration transmissibility of the structure. During the last stage of the research, the vibration damping capacity of the designed frame was assessed using various excitation sources.

Active platforms are another type of anti-vibration platforms, although they perform the same function as passive anti-vibration platforms. Many authors of scientific articles believe that passive anti-vibration platforms are inexpensive and easy to use. However, they have certain limitations, namely than the range of frequencies which can be damped is narrow and that passive anti-vibration platforms cannot be used in some applications. Some people also think that such platforms are ineffective when it comes to damping low-frequency vibrations. In article [14], Nael Nashawati, Chadi Albitar and Alaa Dib presented the results of the analysis of two methods for 
active vibration damping using the RUS Hexapod platform. The RUS (revolute joint, universal joint, spherical joints) anti-vibration platform has six legs. The article presents two methods of vibration control, namely the fuzzy logic method and the adaptive feedforward control method that uses the least-squares multiple-error algorithm. The results were verified using a program created in the Matlab environment. White noise signals were used to simulate vibrations.

XiaoLang Yang et al. wrote an article [15] on the vibration isolation system, based on a flexible Steward platform, which can be used in high-precision space systems. The article focuses on controlling dynamics in six planes. The dynamics equations, based on the pseudo-rigid model, are presented. Flexible joints were taken into account in the model. In addition, a decoupled controller was designed. The lower and upper limits of the frequency range for vibration damping can be regulated.

A platform for damping the low-frequency vibrations of vibration-sensitive devices is presented in article [16]. The platform was designed to prevent the malfunction of optical communication devices, caused by low-frequency seismic excitation. A novel three-dimensional hybrid vibration damping system was used. In order to isolate vibrations horizontally and vertically, the platform was designed as a combination of a rolling isolation system and four threeparameter isolators. A dynamic model of the platform was built and it allowed to reduce the vibration level. The model was validated experimentally. The vibration acceleration was found to have decreased by more than $65 \%$ in the $0-32 \mathrm{~Hz}$ frequency range. The authors discussed the results of the vibration damping efficiency of the active and passive isolators. The results obtained during the research conducted on the platform show that in the vertical direction, to eliminate the amplification effect of the three-parameter isolator and to widen the isolation area, the active damping was used to increase the absorption of vibratory energy.

It is interesting to note that there are many novel vibration damping systems. One of these systems is presented by Xiao Feng and Xingjian Jing in article [17]. The authors built and described a passive anti-vibration system inspired by the human body. It consists of an X-shaped supporting structure (to simulate the legs of the human body) and a rotational element with mass (to mimic the rotational motion of the arms and upper part of the body during walking). Due to the non-linear damping properties of the structure, low transmissibility was achieved in the wide frequency range. The impact of various structural parameters on the vibration isolation efficiency was studied by mathematical modelling. With the proper design of the structure, it was possible to reduce the resonance frequency to about $1 \mathrm{~Hz}$, while maintaining beneficial anti-vibration characteristics.

\subsection{Isolation and damping of vibration in audio/music devices}

The number of companies selling high-quality audio systems is ever-growing. Although there are many different uses for all the vibration damping systems mentioned above, the anti-vibration platform presented in this article is from a scratch implemented to be used with audio devices to maintain the best possible sound quality. The quality of sound depends not only on how it is generated but also on the elimination of any disturbances. The research described in this article were conducted on the anti-vibration platform that can be used in combination with audio/music devices to improve the quality of sound they produce.

Music reproduction qualities, perceived by human senses and the brain are subjective feelings and cannot be measured by any objective measure [18]. In paper [19], E. D. Young et al. presented research on the responses of the squirrel monkey's vestibular neurons to airborne sounds in the frequency range from $50 \mathrm{~Hz}$ to $400 \mathrm{~Hz}$.

The propagation of acoustic waves can be affected by many factors. For example, the type of wood used in the manufacture of musical instruments has a very big impact on the sound character and timbre. Shigeru Yoshikawa presented an acoustic classification of wood for stringed instruments [20]. He indicated that there are two basic types of wood that are used to make musical instruments: wood for soundboards and wood for the back and side plates of instruments. He also 
introduced two parameters in the proposed classification, namely the transmission parameter (product of the propagation speed and the $Q$ value of the longitudinal wave moving along the wood rings) and the anti-vibration parameter (wood density divided by the propagation speed along the wood rings).

There are many signal processing techniques used to reduce impulse noise (unwanted parts of the audio signal) at different stages of sound production. One of these techniques is presented in Ali Awad's article [21]. Sound samples (audio signals), distorted by random impulse noise, were analysed thoroughly in several stages. The aim was to restore the previous quality of the audio signals. At each stage, a vector of different length was used and the degree of similarity between the tested sample and the neighbouring samples was precisely determined for each vector. The tested sample that achieved the maximum degree of similarity with its neighbouring samples at each stage was considered an original sample. Several experiments were performed on different audio sounds to evaluate the performance of the proposed algorithm in terms of peak signal-tonoise ratio (PSNR) and sound quality. The results obtained prove that the new method provides excellent performance compared to other well-known algorithms, especially if the noise is low. The proposed method has proven very effective in removing distorted samples and keeping the original samples intact. In addition, the method is easy to implement and its computational complexity is low.

Another noise reduction algorithm is described in paper [21] by Mehrez Souden et al. The authors presented a multichannel frequency-domain linear filtering algorithm, which can be used to reduce acoustic noise. The performance of this algorithm was assessed, studying many of its aspects. In particular, the authors analysed the effect of coherent and incoherent noise as well as the benefits of using multiple microphones. They also quantified the theoretical gains and losses in terms of speech distortion and noise reduction when using the PWMF (parameterized multichannel non-causal Wiener filter) by establishing new simplified closed-form expressions for three performance measures, namely, the signal distortion index, the noise reduction factor and the output signal-to-noise ratio (SNR). However, signal processing while working very well on test equipment is known to music lovers to introduce its own problems, resulting in a subjective reproduction quality. Therefore, mechanical solutions, like the one presented here, still have a place in modern audio systems as less invasive than signal processing.

\section{Characteristics of the anti-vibration platform for audio/music devices}

The anti-vibration platform for audio devices is shown in Fig. 1. It comprises two parts, which are passive vibration damping systems. It is a structure that uses slate (a mineral that is has been long known for its good mechanical vibration-damping properties).
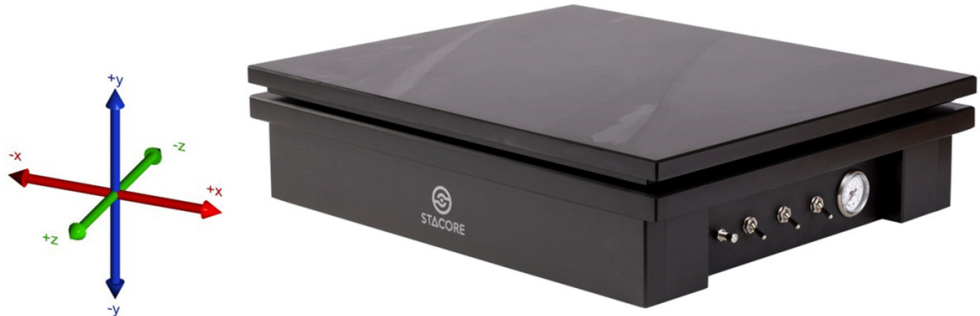

Fig. 1. Stacore advanced anti-vibration platform tested in the article

The first stage of the platform is a pneumatic suspension and consists of four pneumatic elastomer air springs, each with its own air tank - providing pneumatic damping. The air tanks are designed to operate at a maximum pressure of 5 bar. Each spring has a resonance, between $2.5 \mathrm{~Hz}$ and $5 \mathrm{~Hz}$, dependent on the load. The slate case is additionally loaded by a metal plate. It is joined with slate through a viscoelastic layer that also serves as a binder and makes for a 
constrained layer damped sandwich. The second stage of the platform is placed directly above the first one and consists of ball bearings. The bearings are used to isolate transverse vibrations, which are relatively difficult to damp with by the first stage of the platform (the air springs). There are three bearings used, each consisting of a polished deep hardened steel racings and a ball made of tungsten carbide. The total mass of the platform is $94 \mathrm{~kg}$. The upper part of the platform, which rests on the bearings, is a massive constrained layer damped plate, made of metal and slate (weighing $32 \mathrm{~kg}$ ).

\section{Vibration in audio devices}

The principle of operation of the anti-vibration platform is based on damping and isolation of mechanical vibrations. Examples of mechanical waves are sea waves, sound waves and seismic waves. In the process of wave propagation, the process of reversible transformation of potential mechanical energy (pressure energy or stress energy) into kinetic energy is of fundamental importance. During this transformation, some of the energy can be lost; this phenomenon is called wave suppression. The strongest possible damping of unwanted mechanical waves is the desired effect of the anti-vibration platform in question. The vibroisolation of an object means that no vibrations are allowed to interfere with it. Obviously, it is sometimes difficult to clearly separate damping from vibroisolation. It should be noted that the pneumatic system used in the anti-vibration platform performs both vibroisolation (it bends so that what remains above it moves as little as possible) and damping (compression and expansion of air in the tanks, that is to say, the dissipation of energy). The bearings used in the anti-vibration platform have a very low resistance to motion (it means that they are high-precision bearings) and are mainly responsible for the isolation of vibrations and not their damping. This article discusses the research that was conducted on the anti-vibration platform to evaluate its effectiveness of damping and isolation of vibrations.

Both structural vibrations and electroacoustic feedback have an impact on the quality of sound produced by music sets. The structural vibrations of a music set are those vibrations that come from its surroundings (for example, nearby road traffic) and the independent of the musical material. They are relatively harmless for the perceived sound quality. Much more harmful is the electroacoustical feedback. An illustration showing the principle of operation of electroacoustic feedback is presented in Fig. 2. Due to electroacoustic feedback, part of the sound produced by loudspeakers returns in the form of mechanical waves that can cause distortion of newly-played sounds. These disturbances (mechanical waves) can be transmitted to the audio chain both through air (less important) and through the supporting structure (majority of the feedback happens here).
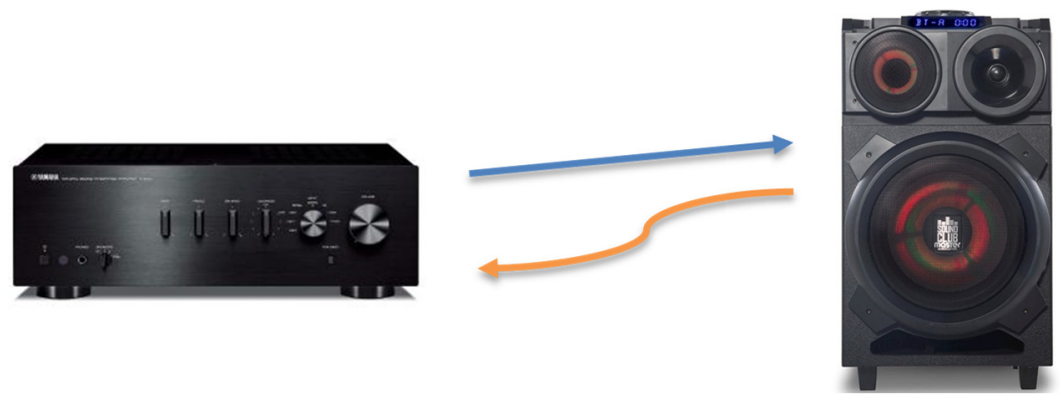

Fig. 2. Principle of operation of electroacoustic feedback

There are many potential parts of chain that can be affected by unwanted vibrations. An exemplary list of some of theme is presented in Fig. 3. Vibration-sensitive equipment are - e.g. turntables, vacuum tubes or capacitors. 


\section{Vibration damping techniques applied to audio devices}

To successfully damp vibrations, they must be decoupled in as many degrees of freedom as possible. Ideally, this should be done using six degrees of freedom (assuming the equipment and the anti-vibration platform are treated as rigid bodies). It is also very important to perform vibration decoupling in a wide frequency range.

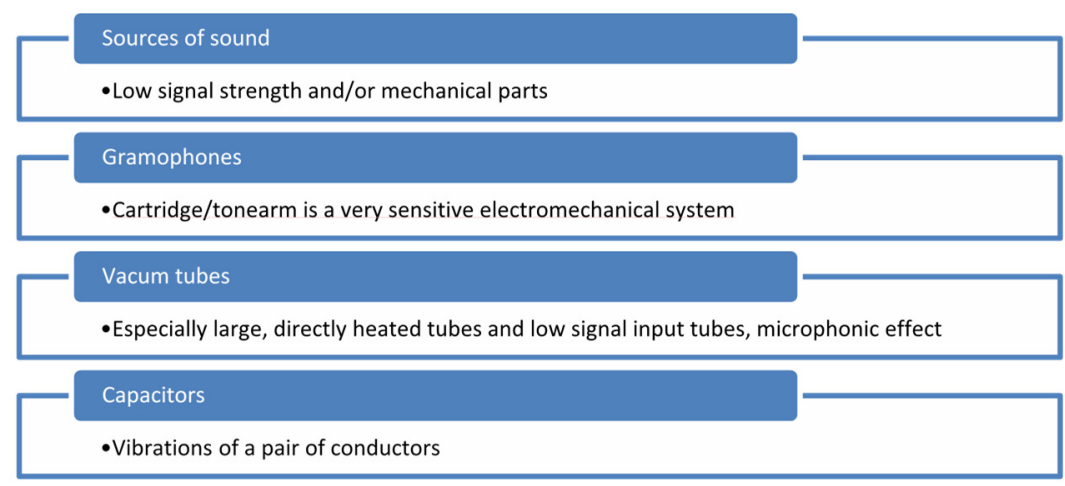

Fig. 3. Vibration sources in audio sets

This is the most important difference of audio-intended platforms from other applications mentioned before, where a wide working frequency range is not needed. Sound waves with frequencies between $20 \mathrm{~Hz}$ and $20,000 \mathrm{~Hz}$ are considered audible by the human ear although both lower and higher frequencies have their influence too, e.g. in a form of wow (low frequencies) or altering sound attach (high frequencies). Anti-vibration platforms should be able to damp the mechanical waves falling within this frequency range, with a possible widening of this range. The basic methods used for vibration damping are presented in Fig. 4. They include vibration decoupling, which means interrupting the vibration path (for example, by placing a vibrating object on an additional foundation), and vibration damping (energy dissipation). The dissipation of mechanical energy can be done in many ways. Usually, vibration damping is the process of converting the energy of unwanted vibrations into heat, which in turn reduces the energy of vibrations. This method is performed, for example, by mechanical friction, using phenomena related to the viscosity of fluids or the blow through of gases.

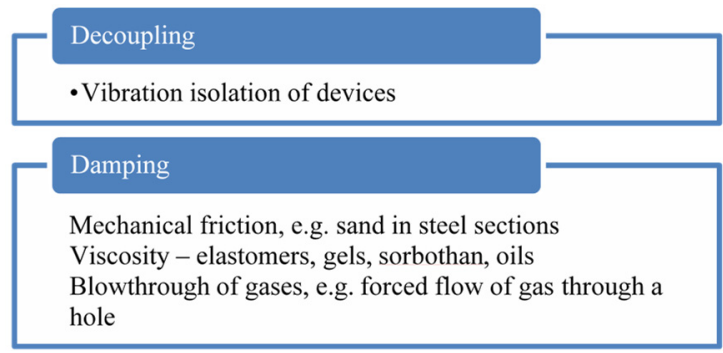

Fig. 4. Vibration reduction methods

For vibration damping, the following methods can be used: passive or active suspensions of various constructions, elastomer layers and rolling bearings.

Elastomers with good damping properties are often used to reduce vibrations. Article [22] by R. K. Luo et al. discusses anti-vibration components of rail vehicles. The authors explain that rubber-to-metal bonded springs can be excellent anti-vibration elements in the suspension systems of these vehicles. They used an integrated design-simulation-testing procedure, which made it possible to combine good vibration damping efficiency with a long service life of the suspension 
components.

The characteristics and properties of the end-of-life car tire rubber are presented in paper [23] by Krzysztof Formela and Józef Haponiuk. They focused on the static and dynamic mechanical properties as well as on the morphology of thermoplastic compositions of low-density polyethene and ground tire rubber. The authors believe that, depending on their composition, the new polymer blends obtained could be applied to car parts or used in the manufacture of road surfaces or parts of anti-vibration systems.

In paper [24], Simi Tang presented the research on the optimisation of a vibration damping method. The main objective of the research conducted was to decrease the mass of the system. Modal analysis and the Ansys software were used to perform vibration damping research. A beam with a fixed support at its both ends was the object of research. Both local damping and global damping were tested. The simulation results obtained showed that the local damping method used made it possible to reduce the mass of the system.

S. Raa Khimi and K. L. Pickering presented a comparison of the dynamic properties of magnetorheological elastomers and anti-vibration rubbers in article [25]. The authors measured the energy dissipated during the hysteresis tests of isotropic and anisotropic MREs (magnetorheological elastomers) containing silane-modified iron sand particles in a natural rubber matrix and compared this value with the results obtained for anti-vibration rubbers.

The dynamic properties of rubber vibration isolators and the anti-vibration research on nanoclay-modified PU/PEL (mPU/polypropylene oxide-polyethylene oxide copolymer with $\mathrm{LiClO}_{4}$ ) blends are presented in article [26]. Analogical research on the ethylene-propylene-diene monomer/nylon 6 blend is described in article [27].

Semin Kwon et al. conducted research on the material that is a complex periodic structure composed of cement concrete embedded with a periodic arrangement of polymer concrete (a mixture of polymeric resin and aggregates) [28]. Compared to cement concrete, the material analysed has superior strength, durability and dynamic characteristics. Experimental research was carried out and the dynamic properties of concrete specimens were measured during vibration experiments performed in the audio frequency range. Using a numerical model, the authors analysed the effect of the periodic structure on the complex concrete beam and it was possible to achieve a significant reduction in vibrations with a small polymer volume fraction.

An analysis of the wear of the components of nuclear steam generators is presented in article [29] by P. L. Ko et al. Wear occurs due to contact at the interface of two contacting bodies. In nuclear power steam generators, high flow rates can induce vibrations of the tubes and cause damage. The mechanisms involved in vibratory wear were analysed. The research results obtained show that distinctly different wear mechanisms and often significantly different wear rates can occur depending on the material combinations.

In article [30], Xiao P. Zhao et al. presented a new kind of self-coupled electrorheological damper and its vibration characteristics. They presented a second-generation damper that has better vibration-damping properties than first-generation dampers. The authors believe that the design method of combining the electrorheological fluid and piezoelectric ceramics could be widely used in acoustic insulation and control systems. The reinforcement of dynamically vulcanised EPDM/PP (ethylene propylene diene monomer/polypropylene) elastomers using fillers is presented in article [31]. An evaluation of the ultimate loading of rubber springs used in engine suspension is presented in article [32] by Kequi Luo et al. In article [33], Jyh-Horng Wu et al. presented the research on the thermal resistance and dynamic damping of thermoplastic composites based on poly (styrene-butadiene-styrene), ester-type polyurethane and ether-type polyurethane materials.

Xiaoning Tang and Xiong Yan wrote an article [34] on the damping properties of fibre-reinforced polymer composites.

The main technical solutions used in the tested anti-vibration platform are listed in Fig. 5. They made it possible to achieve the best possible vibration-damping capacity in the range of audio frequencies. 
-Designed to damp vibrations in the human audible frequency range and below

Pneumatic base

-Damped pneumatic suspension

Broadband vibration damping

- Large mass of the platform as a part of the damping mechanism

-Amorphous slate with very good vibration-damping properties was used

Two levels of decoupling

- The lower level consists of air springs

-The upper level uses rolling bearings

Fig. 5. Characteristics of anti-vibration platform

\section{Experimental research}

The experimental research aimed at checking the vibration-damping capacity of the anti-vibration platform for audio devices. The research was conducted with the manufacture of the anti-vibration platform and is part of the development of this line of products. In the future, results from experimental studies can be used to develop an even more efficient anti-vibration platforms or another type of vibration control devices. The next part of the article presents the measuring apparatus used, the configuration of the test rig and the results of the measurements.

\subsection{Test rig configuration}

A scheme of the test rig used to verify the vibration-damping efficiency of the anti-vibration platform is presented in Fig. 6. The platform was placed on a rigid plate that was supported by four-footers connected to pneumatic vibroisolators. The plate was suspended using four elastic ropes attached to the plate corners (on their one side) and to the supporting structure (on the other side of the ropes). An electromagnetic vibration exciter (TIRA GmbH S 51110) was placed under the plate and set at an angle of 45 degrees to its lower surface.

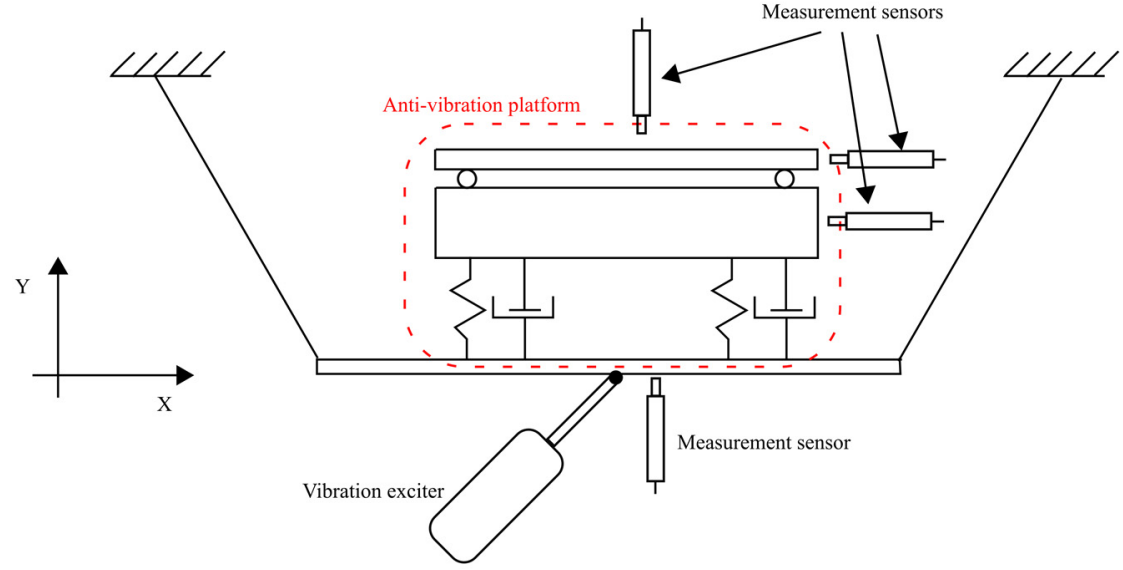

Fig. 6. Measurement scheme

The measurement of displacements was performed using laser displacement sensors (Keyence LK-H050), as shown in Fig. 7(a). The first sensor was located near the vibration exciter and it was 
used to measure displacements in the direction perpendicular to the lateral surface of the plate. The second sensor measured the displacements of the plate in the direction perpendicular to its upper surface. Based on the difference in the amplitude of the signals recorded by these sensors, the efficiency of the vibration damping in the vertical direction was determined. The second sensor arrangement was used to measure the effectiveness of vibration damping in the horizontal direction. In this case, one of the sensors pointed towards the base and the second one towards the upper plate, as shown in Fig. 7(a). The difference in the vibration amplitude recorded by these sensors was used to determine the effectiveness of vibration damping of the anti-vibration platform in the horizontal direction.

Fig. 7(b) shows a photo of the data acquisition system (SCADAS Mobile by LMS International), which was used in the experimental research. It was used for collecting data recorded by laser sensors and controlling the vibration exciter.

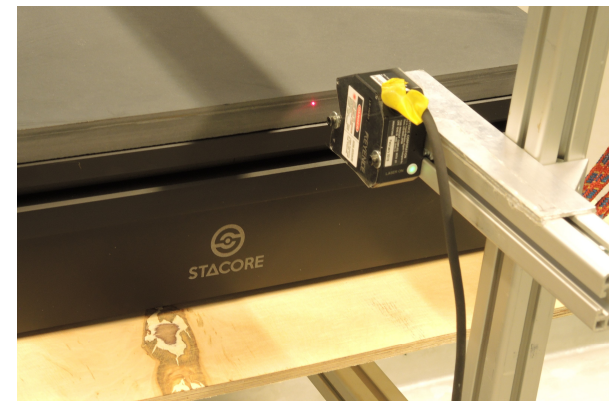

a)

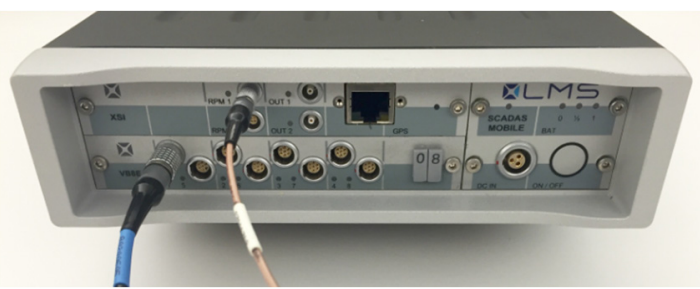

b)

Fig. 7. a) Displacement measurement of upper plate, b) data acquisition module (SCADAS mobile)

A photo showing the tested anti-vibration platform suspended on ropes and the vibration exciter (placed on the floor) is demonstrated in Fig. 8. This photo also shows the laser sensor that was used to measure vibration in the vertical direction. The first laser sensor is located near the vibration exciter and the second one is visible at the top of the photo. The platform was additionally loaded with a $10 \mathrm{~kg}$ slate plate to bring it closer to real-life operation.

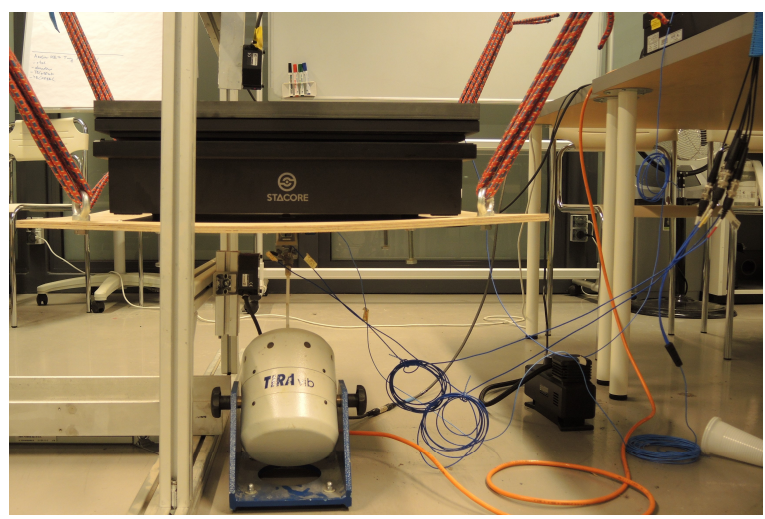

Fig. 8. Photo that was taken during the experimental research

\subsection{Results of the experimental research}

The vibration exciter was connected to the plate (on which the anti-vibration platform was resting) by means of a flexible tension member, at the end of which the impedance head was mounted. The head was used to measure the excitation force and the vibration acceleration. The electromagnetic vibration exciter was used to excite the vibration of the tested platform. During 
the experiment, the frequency of the excitation force was changed and after that, there was a period of stable operation, which lasted 8 seconds. The graph showing the excitation force recorded by the force sensor is shown in Fig. 9(b). Let's take a look at this graph. It can be seen that the force was almost constant during the first eight seconds of the measurement (for an excitation frequency of $8 \mathrm{~Hz}$ ). The graph showing the displacement registered (during the same measurement) by the laser sensor placed near the exciter is presented in Fig. 9(a). After starting the excitation by means of a vibration exciter (which simulated the noisy environment), the anti-vibration platform was continuously damping the vibration, which is visible in a decrease of the vibration amplitude of the element that was placed on top of the anti-vibration platform. We can see that in the beginning, this process was not stationary. Immediately after switching on the exciter, the vibration amplitude increased and then was decreasing over time to reach constant and repeatable values. After taking a look at this graph, it is clear that it took approximately 8 seconds for the initial vibration of the platform to be stable. During the experiment, a constant-amplitude force was applied and its frequency was modified.
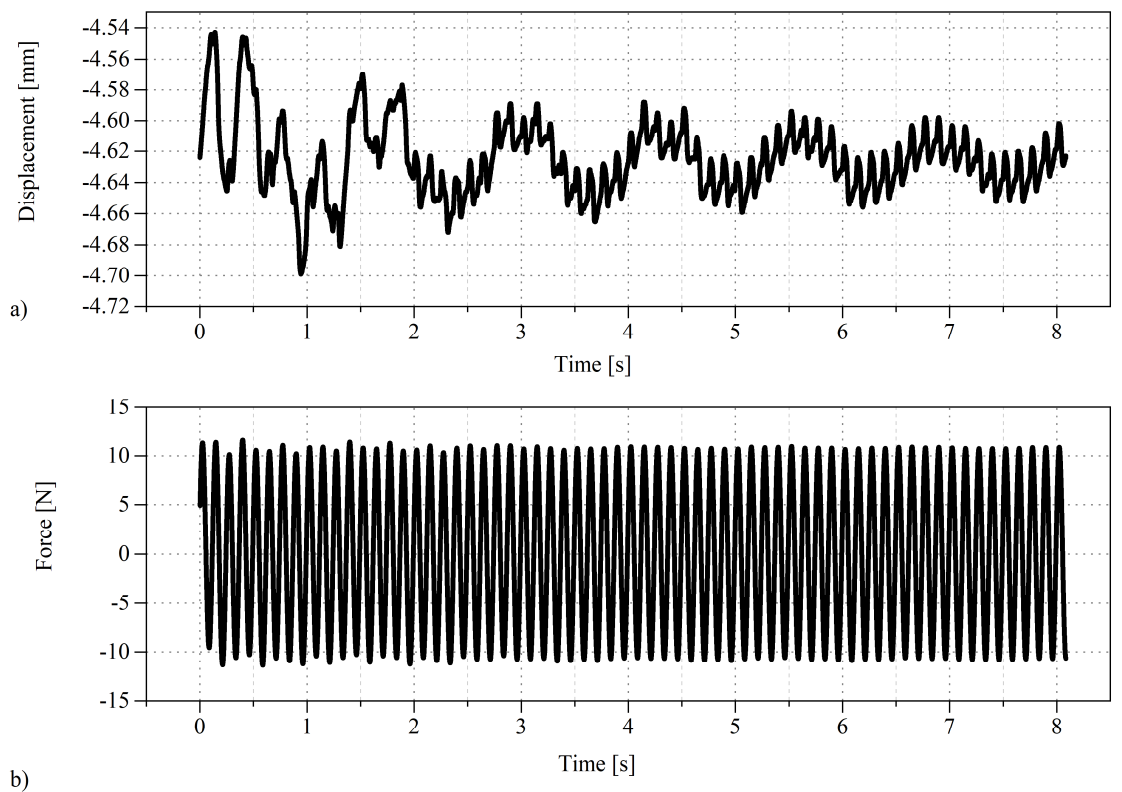

Fig. 9. a) Input signal registered by the laser displacement sensor located close to the vibration exciter, b) force generated by the vibration exciter

The signals recorded for the $5.0 \mathrm{~s}$ to $8.0 \mathrm{~s}$ time interval, at various excitation frequencies $(0.5 \mathrm{~Hz}, 2 \mathrm{~Hz}, 4 \mathrm{~Hz}, 8 \mathrm{~Hz}$ and $15 \mathrm{~Hz})$, are presented in Fig. 10. In the cases where the excitation frequency is close or equal to one of the eigenfrequencies of the anti-vibration platform, the stabilisation time was a little bit longer than in the other cases.

When increasing the frequency, it was normal for the amplitude of the forced vibration to be lower than the one visible on the graphs. For example, when the excitation force was $0.5 \mathrm{~Hz}$, the peak-to-peak (pk-pk) amplitude in $Y$ direction was almost $3 \mathrm{~mm}$. When excitation frequency was increased to $4 \mathrm{~Hz}$, the amplitude was $0.1 \mathrm{~mm}$. When the exciter was set to excite the structure with a frequency of $15 \mathrm{~Hz}$, the amplitude was only $0.01 \mathrm{~mm}$. In some cases (for example, output $Y$ direction for $8 \mathrm{~Hz}$ ), we can clearly see the combination of two frequencies. The first part of the signal is connected to the excitation force $(8 \mathrm{~Hz})$ and the second part of the signal is connected to the eigenfrequencies of the anti-vibration platform (about $0.5 \mathrm{~Hz}$ ): 
Transmissibility $=\frac{\text { Amplitude }_{\text {input }}}{\text { Amplitude }_{\text {output }}}$.

Fig. 11 summarizes the results of the experimental research conducted on the anti-vibration platform intended to be used with audio devices. The graph presents the efficiency of vibration damping of the platform. The $x$-axis indicates the frequency of the excitation force generated by the vibration exciter and the $y$-axis indicates the ratio of the amplitude of the input signal to the amplitude of the output signal (see Eq. (1)). No optimization algorithm was used to calculate the results. The black line shows the effectiveness of vibration damping in the direction perpendicular to the plane of the anti-vibration platform ( $y$-direction in Fig. 1) whereas the red line shows the results obtained for the direction perpendicular to this direction.

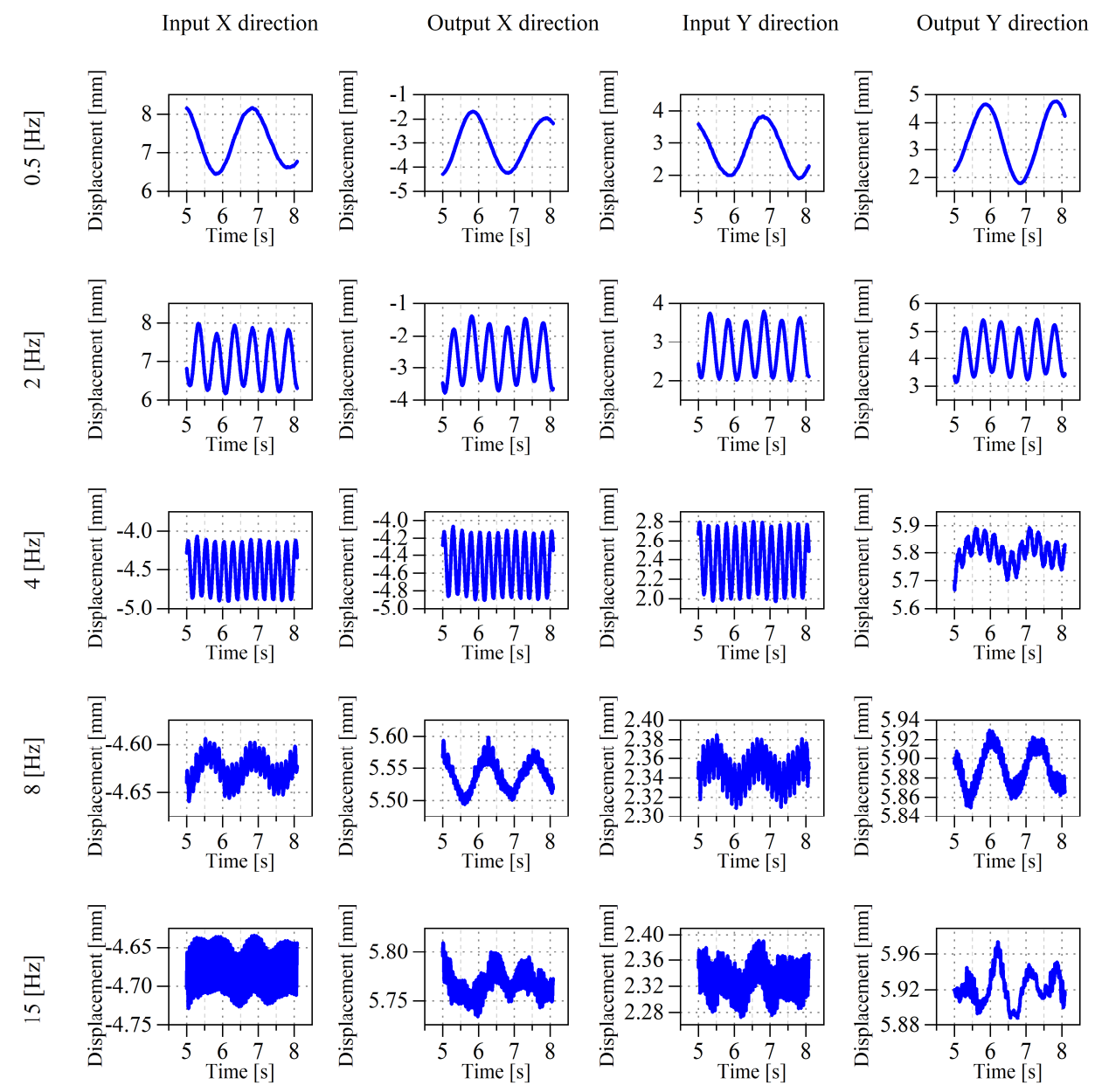

Fig. 10. Signals registered during the experimental research conducted on the anti-vibration platform intended for use with audio devices

A value of 1 on the $y$-axis means that the vibration transmitted through the platform had the same amplitude as the vibration amplitude of the excitation force. Values greater than 1 mean that there was the amplification of vibration while values lesser than 1 mean that there was vibration damping. In the vertical direction, the resonance frequency was about $4 \mathrm{~Hz}$, and in the horizontal direction, it was about $1 \mathrm{~Hz}$. One can also observe the anti-resonance phenomenon or the area in which the vibration damping is weaker (around a frequency of $4 \mathrm{~Hz}$ ). This was most likely as a 
consequence of coupling the vibration in this plane with the vibration in the vertical plane where the resonance frequency was approximately $4 \mathrm{~Hz}$. In general, it can be said that the higher the excitation frequency, the better the isolation of the platform. At frequencies greater than $8 \mathrm{~Hz}$, the percentage of isolation in the vertical and horizontal directions appears similar and the damping increases simultaneously. The vibration signal below $8 \mathrm{~Hz}$ has relatively the highest values because of the resonances of the structure (we can assume that the good damping starts already at $2.5 \mathrm{~Hz}$ in the horizontal plane and $7 \mathrm{~Hz}$ in the vertical plane). The resonances occurred mainly because of the pneumatic suspension. By altering the pressure, the pneumatic suspension resonance can be in principle tuned in some limits but this was not tested in the experiment, operating the platform at the lowest possible frequency (giving the lowest resonance for a given load. For "music lovers", the most important thing is to obtain the lowest transmissibility in the frequency band that is connected to the audible frequencies. Most often, we assumed that these were the frequencies within the range from $20 \mathrm{~Hz}$ to $20 \mathrm{kHz}$. In these cases, the most appreciated results are to move the resonance frequencies (the frequency regions in which there is amplification of the signal) as "far" as possible from the frequencies that are desired to be the most damped. In this case, it can be assumed that good damping is at frequencies starting from $2.5-7 \mathrm{~Hz}$ (depending on the plane) and that the goal has been achieved (in the range of frequencies analysed).

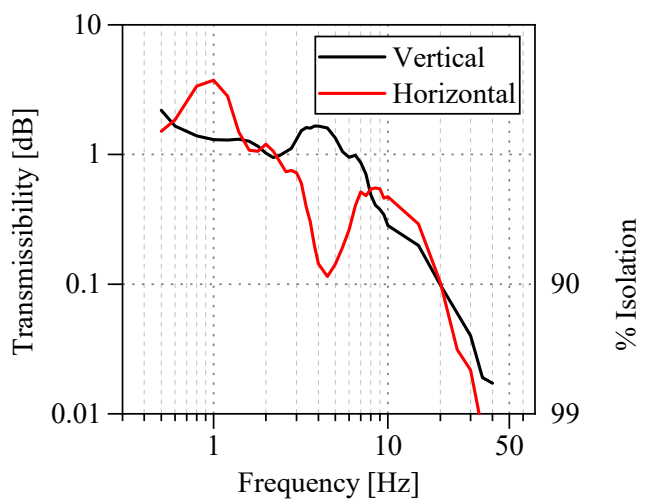

Fig. 11. Transmissibility of anti-vibration platform

\section{Conclusions}

The article discusses the effectiveness of vibration damping of the anti-vibration platform intended for use in combination with audio/music equipment. The research was conducted on behalf of the manufacturer and together with him. Therefore, the article gives many details of the design of the special-purpose anti-vibration platform. It has to deal with both subsonic frequencies (below $20 \mathrm{~Hz}$ ), which modulate the sound, as well as the directly audible ones in the range $20 \mathrm{~Hz}-20 \mathrm{kHz}$. And the platform presented herein was specifically designed for this purpose. The unique feature of this platform is the combination of several damping mechanisms, including air dampers, ball bearing and the casing made of amorphous slate, further damped using constrained layer technique.

In this work we have examined only low frequency behavior of the platform due to the different test techniques and equipment needed for higher frequencies. The experimental research was carried out on a specially prepared test rig, where the anti-vibration platform was suspended on flexible lines. The vibration excitation of the platform was performed using an electromagnetic exciter. Next, displacements near the exciter and at the upper part of the plate were measured; the objects that were put on the plate were isolated from vibration. The vibration damping efficiency was evaluated on the basis of the ratio of the vibration amplitude of the lower part of the antivibration platform to the vibration amplitude of its upper part. In the article are included not only 
the results of measurements but also the intermediate results that include the time signal of the exciter. On this signal is visible the time needed to stabilize the displacements and force. It also includes the signals measured by four displacement sensors for five excitation frequencies. The results of the experimental research conducted are proof that the platform was able to damp vibrations appropriately in the wide frequency range, which also includes the frequencies of acoustic waves. In the vertical direction (perpendicular to the plane, there is good vibration damping at frequencies slightly above $7 \mathrm{~Hz}$ whereas in the horizontal direction there is already good vibration damping at frequencies slightly above $2.5 \mathrm{~Hz}$ (in the range of frequencies analysed).

Even though there are no scientific articles describing the research on anti-vibration platforms for audio equipment, the results obtained can be compared with the data available for anti-vibration platforms of a different type. The low-frequency vibration damping platform [16], which should be used with vibration-sensitive devices, is an example that can be referred to here. As in the analysed platform, isolators in combination with electromagnetic actuators were used for the damping of vertical vibrations. In addition, ball bearings were used to damp horizontal vibrations. The additional active damping of vibrations (using an electromagnetic actuator) was only an option. The authors presented the results of vibration damping with and without the active damping system. With regard to the vibration damping platform discussed in this paper, a resonance frequency (about $10.5 \mathrm{~Hz}$ ) can be observed when only passive vibration reduction is used. But when both passive and active vibration reduction techniques are used, no resonance frequency occurs. The platform discussed here has better vibration-damping characteristics than the one presented in article [16] because the vibrations of the wider range of "audio" frequencies can be damped.

\section{References}

[1] Clemente P., Rinaldis D. Protection of a monumental building against traffic-induced vibrations. Soil Dynamics and Earthquake Engineering, Vol. 17, Issue 5, 1998, p. 289-296.

[2] Griffin M. J. Measurement, evaluation, and assessment of occupational exposures to hand-transmitted vibration. Occupational and Environmental Medicine, Vol. 54, Issue 2, 1997, p. 73-89.

[3] Żywica G., Breńkacz L., Bagiński P. Interactions in the rotor-bearings-support structure system of the multi-stage ORC microturbine. Journal of Vibration Engineering and Technologies, Vol. 6, Issue 5, 2018, p. 369-377.

[4] Bovenzi M., Alessandrini B., Mancini R., Cannavà M. G., Centi L. A prospective study of the cold response of digital vessels in forestry workers exposed to saw vibration. International Archives of Occupational and Environmental Health, Vol. 71, Issue 7, 1998, p. 493-498.

[5] Krajnak K., Waugh S., Johnson C., Miller R. G., Welcome D., et al. Antivibration gloves: effects on vascular and sensorineural function, an animal model. Journal of Toxicology and Environmental Health, Part A, Vol. 78, Issue 9, 2015, p. 571-582.

[6] Kyu-Jung K., Il Kyu H. Shock-absorbing effects of various padding conditions in improving efficacy of wrist guards. Journal of Sports Science and Medicine, Vol. 3, Issue 1, 2004, p. 23.

[7] Chiang H. H., Chen Y. L., Hsu K. C. Optimized sensorless antivibration control for semiactive suspensions with cosimulation analysis. IEEE/ASME Transactions on Mechatronics, Vol. 20, Issue 4, 2015, p. 1898-1911.

[8] Wang B., Xu W., Zhao M., Wu H. Antivibration pipeline-filtering algorithm for maritime small target detection. Optical Engineering, Vol. 53, Issue 11, 2014, p. 113109.

[9] Breńkacz L., Żywica G., Drosińska-Komor M. The experimental identification of the dynamic coefficients of two hydrodynamic journal bearings operating at constant rotational speed and under nonlinear conditions. Polish Maritime Research, Vol. 24, Issue 96, 2017, p. 108-115.

[10] Breńkacz L., Żywica G. Comparison of experimentally and numerically determined dynamic coefficients of the hydrodynamic slide bearings operating in the nonlinear rotating system. Turbomachinery Technical Conference and Exposition, USA, 2017.

[11] Deng W., Zhao H., Zou L., Li G., Yang X., Wu D. A novel collaborative optimization algorithm in solving complex optimization problems. Soft Computing, Vol. 21, Issue 15, 2017, p. 4387-4398.

[12] Deng W., Xu J., Zhao H. An improved ant colony optimization algorithm based on hybrid strategies for scheduling problem. IEEE Access, Vol. 7, 2019, p. 20281-20292. 
[13] Wen X., Li W., Fang Y., Song C., Zhang J. Design and Vibration Isolation Performance of TrussType CFRP Raft Frame. Shock and Vibration, Vol. 2019, 2019, p. 4281958.

[14] Nashawati N., Albitar C., Dib A. The evaluation of two methods for active vibration damping using RUS Hexapod platform. Transactions of the Institute of Measurement and Control, Vol. 41, Issue 5, 2019, p. 1207-1215.

[15] Yang X. L., Wu H. T., Chen B., Kang S. Z., Cheng S. L. Dynamic modeling and decoupled control of a flexible Stewart platform for vibration isolation. Journal of Sound and Vibration, Vol. 439, 2019, p. 398-412.

[16] Xie X., Diao J., Xu Y., Zhang Z. Performance of a low-frequency hybrid vibration isolation platform for vibration-sensitive devices. Journal of Low Frequency Noise, Vibration and Active Control, Vol. 37, Issue 4, 2018, p. 1164-1175.

[17] Feng X., Jing X. Human body inspired vibration isolation: Beneficial nonlinear stiffness, nonlinear damping and nonlinear inertia. Mechanical Systems and Signal Processing, Vol. 117, 2019, p. 786-812.

[18] Baumgarte F., Faller C. Binaural cue coding - part I: psychoacoustic fundamentals and design principles. IEEE Transactions on Speech and Audio Processing, Vol. 11, Issue 6, 2003, p. 509-519.

[19] Young E. D., Fernández C., Goldberg J. M. Responses of squirrel monkey vestibular neurons to audio-frequency sound and head vibration. Acta Oto-Laryngologica, Vol. 84, Issues 1-6, 1977, p. 352-360.

[20] Yoshikawa S. Acoustical classification of woods for string instruments. The Journal of the Acoustical Society of America, Vol. 122, Issue 1, 2007, p. 568-573.

[21] Souden M., Benesty J., Affes S. On optimal frequency-domain multichannel linear filtering for noise reduction. IEEE Transactions on Audio, Speech and Language Processing, Vol. 18, Issue 2, 2010, p. 260-276.

[22] Luo R. K., Cook P. W., Wu W. X., Mortel W. J. Fatigue design of rubber springs used in rail vehicle suspensions. Proceedings of the Institution of Mechanical Engineers, Part F: Journal of Rail and Rapid Transit, Vol. 217, Issue 3, 2003, p. 237-240.

[23] Formela K., Haponiuk J. Characterization and properties of LDPE/(ground tire rubber)/crosslinked butyl rubber blends. Journal of Vinyl and Additive Technology, Vol. 20, Issue 4, 2014, p. 237-242.

[24] Tang S. Research on optimization design of local damping vibration reduction. IOP Conference Series: Materials Science and Engineering, Vol. 472, 2019, p. 012058.

[25] Khimi S. R., Pickering K. L. Comparison of dynamic properties of magnetorheological elastomers with existing antivibration rubbers. Composites Part B: Engineering, Vol. 83, 2015, p. 175-183.

[26] Chiu H. T., Wu J. H., Shong Z. J. Dynamic properties of rubber vibration isolators and antivibration performance of nanoclay-modified PU/PEL blends system. Polymer Engineering and Science, Vol. 45, Issue 4, 2005, p. 539-548.

[27] Wu J.-H., Li C.-H., Chiu H.-T., Shong Z.-J. Dynamic properties of rubber vibration isolators and antivibration performance of ethylene-propylene-diene monomer/nylon 6 blend systems. Journal of Applied Polymer Science, Vol. 108, Issue 6, 2008, p. 4114-4121.

[28] Kwon S., Ahn S., Koh H. I., Park J. Polymer concrete periodic meta-structure to enhance damping for vibration reduction. Composite Structures, Vol. 215, 2019, p. 385-390.

[29] Ko P. L., Taponat M.-C., Zbinden M. Wear studies of materials for tubes and antivibration bars in nuclear steam generators. Journal of Pressure Vessel Technology, Vol. 118, Issue 3, 2008, p. 287.

[30] Zhao X. P., Liu S., Tang H., Yin J. B., Luo C. R. A new kind of self-coupled electrorheological damper and its vibration character. Journal of Intelligent Material Systems and Structures, Vol. 16, Issue 1, 2005, p. 57-65.

[31] Wu J. H., Li C. H., Chiu H. T., Shong Z. J., Tsai P. A. Reinforcement of dynamically vulcanized EPDM/PP elastomers using organoclay fillers: Dynamic properties of rubber vibration isolators and antivibration performance. Journal of Thermoplastic Composite Materials, Vol. 22, Issue 5, 2009, p. 503-517.

[32] Luo R. K., Wu W. X., Mortel W. J. Ultimate loading evaluation of rubber springs used in engine suspension. Proceedings of the Institution of Mechanical Engineers, Part L: Journal of Materials: Design and Applications, Vol. 217, Issue 4, 2003, p. 287-290.

[33] Wu J. H., Li C. H., Wu Y. T., Leu M. T., Tsai Y. Thermal resistance and dynamic damping properties of poly (styrene-butadiene-styrene)/thermoplastic polyurethane composites elastomer material. Composites Science and Technology, Vol. 70, Issue 8, 2010, p. 1258-1264.

[34] Tang X., Yan X. A review on the damping properties of fiber reinforced polymer composites. Journal of Industrial Textiles, 2018, https://doi.org/10.1177/1528083718795914. 


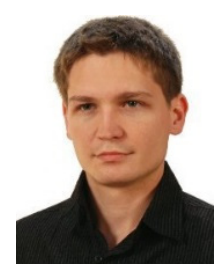

Lukasz Breńkacz, Ph.D., Eng. He works at the Institute of Fluid Flow Machinery, Polish Academy of Sciences, in Gdansk as a research associate. His current research interests include designing of machinery, analysis of bearing systems, computer simulations and experimental diagnostics of rotating machinery. Member of the Polish Society of Technical Diagnostics and ASME, an expert in the Association of Polish Mechanical Engineers and Technicians.

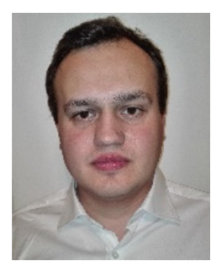

Pawel Bagiński is in the process of writing his Ph.D. dissertation. He works as a scientific assistant at the Institute of Fluid-Flow Machinery in Gdansk. He is currently involved in research on foil bearings as well as dynamic properties of structures and fluid-flow machinery and these topics are closely linked to his scientific interests.

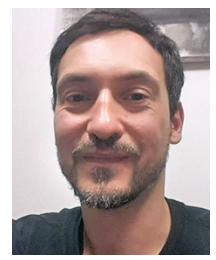

Jaroslaw Korbicz, physicists working with quantum information science. Currently, he is an Associate Professor at the Center of Theoretical Physics of the Polish Academy of Sciences. In 2000, received a diploma from the University of Warsaw (Faculty of Physics). He defended his doctoral dissertation at Hannover University in 2006. In 2017, qualified as an Assistant Professor at the University of Gdańsk. In 2015, together with his friend Bogdan Stasiak, M.Sc. Eng., who is a specialist in the field of mechanical engineering, he established the Stacore company. The company offers specialised solutions for high-end audio equipment, intended for vibration isolation and damping. 